\title{
The behaviour of Li abundance in the stars of different metallicities
}

\author{
T.V. Mishenina ${ }^{1}$ and C. Soubiran ${ }^{2}$ \\ ${ }^{1}$ Odessa Astronomical Observatory, Park Shevchenko, 65014, Odessa, Ukraine \\ email: tamar@deneb.odessa.ua \\ ${ }^{2}$ Observatoire Aquitain des Sciences de l'Univers, CNRS UMR 5804,BP 89, \\ 33270 Floirac, France
}

\begin{abstract}
Using the method of spectral synthesis we derived the abundances of $\mathrm{Li}$ in the atmospheres of 100 stars in the range of metallicities $-3<[\mathrm{Fe} / \mathrm{H}]<0.2$. The investigated spectra are part of the library collected at the Haute Provence Observatory and they were obtained with the $193 \mathrm{~cm}$ telescope equipped with ELODIE spectrometer $(\mathrm{R}=42000)$. For the metal-poor dwarfs, in the "Spite plateau" region $\left([\mathrm{Fe} / \mathrm{H}]<-1.7, T_{\text {eff }}>5700 \mathrm{~K}\right)$ we obtain $\log \mathrm{A}(\mathrm{Li})=2.30 \pm 0.05$, which is in a good agreement with the results of other authors. Our values of $\mathrm{Li}$ abundances do not indicate any trends either with $T_{\text {eff }}$ or with $[\mathrm{Fe} / \mathrm{H}]$. The "plateau" is also traced in the metallicity range $-0.7<[\mathrm{Fe} / \mathrm{H}]<-0.3$. The behavior of the lithium abundance for stars with $[\mathrm{Fe} / \mathrm{H}]>-1.7$ and $T_{\text {eff }}<5700 \mathrm{~K}$ shows a depleting mechanism in these stars and the growth of its efficiency with an increase of the metallicity. The "lithium plateau" was also found for thick disk dwarfs with $T_{\text {eff }}>5800 \mathrm{~K}$.
\end{abstract}

Keywords. Stars: evolution, stars: abundances

\section{Introduction}

The distinguishing feature of the $\mathrm{Li}$ abundance behavior in metal-poor stars is that the halo dwarfs at $[\mathrm{Fe} / \mathrm{H}]<-1.5$ show almost the same $\mathrm{Li}$ abundance $\log \mathrm{A}(\mathrm{Li})=2.1$ (Spite \& Spite, 1982). Spite \& Spite (1982) suppose that the obtained value is the primordial abundance of Li. There is a large discussion of the following aspects: 1) the dispersion of the Li plateau values; 2 ) the trends of the $\mathrm{Li}$ abundance with $[\mathrm{Fe} / \mathrm{H}]$ and $T_{\text {eff }} ; 3$ ) what physical processes of the transport and/or depletion might be acting in the plateau stars; 4) what is the value of the primordial lithium abundance? (Deliyannis, Pinsonneault \& Duncan (1993); Gratton, Sneden, Carretta E. \& Bragaglia (2000); Ryan, Norris \& Beers (1999); Salaris \& Weiss (2001); Thorburn (1994); Vauclair \& Charbonnel (1998) etc.).

\section{Observations, parameters and Li abundance determinations}

The spectra were obtained with the $193 \mathrm{~cm}$ telescope (OHP) equipped with ELODIE spectrometer $(\mathrm{R}=42000)$ (Soubiran, Katz, \& Cayrel 1998). The spectral range is 4400 $6800 \AA \AA, \mathrm{S} / \mathrm{N}>100$. The effective temperature $T_{\text {eff }}$ were determined from the fitting of the far wings of $\mathrm{H}_{\alpha}$ lines, $\log \mathrm{g}$ values were obtained from ionization balance for iron, and $V_{\mathrm{t}}$ values were found by requiring of the iron abundances obtained from the given line of Fe I to be independent on its equivalent widths EW. The Li abundances in program stars were obtained by the STARSP LTE spectral synthesis code by Tsymbal (1996). A list of atomic and molecular lines was taken from (Mishenina \& Tsymbal 1997). 

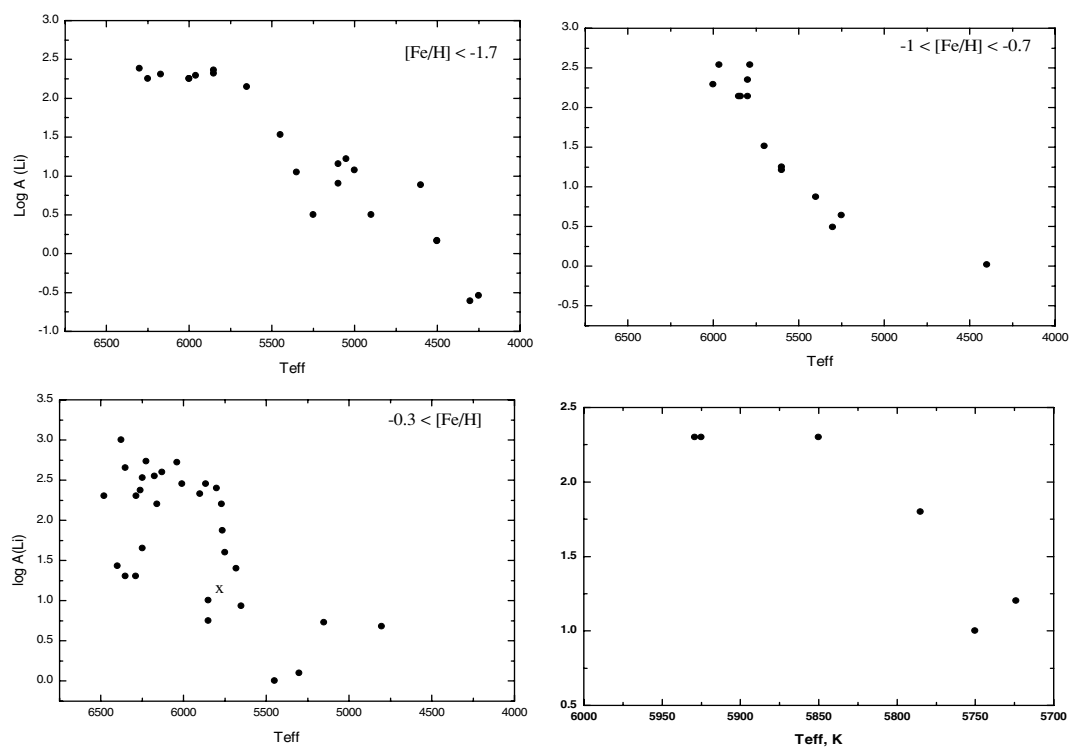

Figure 1. $\log \mathrm{A}(\mathrm{Li})$ vs. $T_{\text {eff }}$ for different metallicity stars (thick disk stars at bottom right)

\section{Results and conclusions}

The dependence of $\mathrm{Li}$ behavior on the temperature for the different metallicities is shown in Fig. 1. As one can see from Fig. 1 (top left), the most metal-poor stars show a very narrow plateau with the scatter of only \pm 0.058 dex. In a slightly more metal rich groups (top right), the plateau is observed too, but with a larger scatter $( \pm 0.17 \mathrm{dex})$, the values of the plateau is similar $(2.31 \mathrm{dex})$. The plateau extends up to $[\mathrm{Fe} / \mathrm{H}]=-0.3$. The behavior of the $\mathrm{Li}$ abundance with temperature for different metallicity groups indicates that efficiency of the transport mechanisms increases with the metallicity. We confirm the bimodal distribution of Li for stars around solar $[\mathrm{Fe} / \mathrm{H}]$ and $T_{\text {eff }}>5600 \mathrm{~K}$ (bottom left, the cross marks the Sun).

We found also the "lithium plateau" (bottom right) for the thick disk dwarfs $\left(T_{\text {eff }}>5800 \mathrm{~K}\right)$, that we have selected on kinematical criterion. The behavior of the lithium abundance in stars with $T_{\text {eff }}<5600 \mathrm{~K}$ supports the existence of some depleting mechanism.

\section{Acknowledgements}

We would like to acknowledge Dr. V. Kovtyukh for some comments and we also thank the organisers for the financial support by IAU grant.

\section{References}

Deliyannis, C.P., Pinsonneault, M.H. \& Duncan, D.K. 1993, ApJ, 414, 740

Gratton, R.G., Sneden, C., Carretta, E. \& Bragaglia, A. 2000, A\&A, 354, 1

Mishenina, T.V. \& Tsymbal, V.V. 1997, Pis'ma v AZh 23, 693

Ryan, S.G., Norris, J.E. \& Beers, T.C. 1999, ApJ, 523, 654

Salaris, M. \& Weiss, A. 2001, A\& $A, 376,955$

Spite, F. \& Spite, M. 1982, A\&A 115, 357

Soubiran, C., Katz, D., \& Cayrel, R. 1998, A\& $A S$, 133, 221

Thorburn, J.A. 1994, ApJ, 421, 318

Tsymbal, V.V. 1996, ASP Conf. Ser., 108, 198

Vauclair, S. \& Charbonnel, C. 1998, ApJ, 502, 372 